\title{
Ultrasound-guided percutaneous microwave ablation for benign breast lesions: evaluated by contrast-enhanced ultrasound combined with magnetic resonance imaging
}

\author{
Wei Zhang ${ }^{1}$, Jian-Min Li ${ }^{1}$, Wen He ${ }^{2}$, Xiao-Ming Pan $^{3}$, Zhan-Qiang Jin ${ }^{2}$, Ting Liang ${ }^{1}$, Hui Zhang ${ }^{3}$ \\ ${ }^{1}$ Department of Ultrasound, The 3th Affiliated Hospital of Guangxi Medical University, Nanning 530021, China; ${ }^{2}$ Department of Ultrasound, \\ Beijing Tiantan Hospital, Capital Medical University, Beijing 100050, China; ${ }^{3}$ Department of Thyroid Surgery, The 3th Affiliated Hospital of \\ Guangxi Medical University, Nanning 530021, China \\ Contributions: (I) Concept and design: W He, W Zhang; (II) Administrative Support: JM Li; (III) Provision of the study materials and patients: \\ XM Pan, H Zhang; (IV) Collection and assembly of data: ZQ Jin, T Liang; (V) Data analysis and interpretation: W He, W Zhang, ZQ Jin; (VI) \\ Manuscript writing: W He, ZQ Jin; (VII) Final approval of manuscript: All authors. \\ Correspondence to: Wen He, Professor. Department of Ultrasound, Beijing Tiantan Hospital, Capital Medical University, No. 6 Tiantan Xili, \\ Dongcheng District, Beijing 100050, China. Email: ttyyus@sina.com.
}

\begin{abstract}
Background: Minimally invasive ablative techniques in the treatment of breast tumor has become popularly in recent years.

Methods: We analyzed gray-scale and contrast-enhanced ultrasound (CEUS) features of 205 microwave ablated breast benign lesions from 182 consecutive patients, compared with magnetic resonance imaging (MRI) and histopathology findings. The follow-up was implemented at 3, 6 and 12 months after the ablation treatment.

Results: Before the MWA, the mean of largest diameter and volume of the lesions were $14.41 \pm 6.54$ and $3,224 \pm 961 \mathrm{~mm}^{3}$, respectively. However, those of the lesions respectively were $8.48 \pm 6.30$ and $2,116 \pm 732 \mathrm{~mm}^{3}$ one year after the treatment. The longest diameter and the volume of the ablative lesions were gradually decreased 3, 6 or 12 months after the MWA. 44 (/205, 21.5\%) ablative lesions were disappeared one year after the MWA. One hundred and forty-two (/205, 69.3\%) ablative lesions presented a hypoechoic halo surrounding it on gray-scale US after the MWA. The success rate of the MWA treatment in the benign breast lesion was $87.32 \%$ and $82.93 \%$ evaluated by CEUS and enhanced MRI, respectively. During the ablation, no patient had serious complications, such as hemorrhage, serious pain and fat necrosis, etc.

Conclusions: Microwave ablation was a safe and efficient method in the treatment of the benign breast tumors. CEUS and enhanced MRI could accurately assess whether the MWA treatment is effective.
\end{abstract}

Keywords: Breast benign tumor; microwave ablation (MWA); contrast-enhanced ultrasound (CEUS); magnetic resonance imaging (MRI)

Submitted Aug 05, 2017. Accepted for publication Sep 07, 2017.

doi: $10.21037 /$ jtd.2017.09.132

View this article at: http://dx.doi.org/10.21037/jtd.2017.09.132

\section{Introduction}

Breast tumor is the most commonly diagnosed tumor in women, whose treatment has changed considerably owing to the difference of clinical presentation, patients' subjective awareness and cosmesis concerns, as well as increased detection rate of small tumors (1). For these reasons, less invasive methodologies, such as radiotherapy, Mammotome system and percutaneous ablation have been increasingly used for the treatment of breast tumors, especially benign lesions. Percutaneous ablation is a minimally invasive method for the treatment of breast tumors guided by using ultrasound (US) or magnetic resonance imaging (MRI), 
which has intrigued extensive attention as a possible firstline treatment to substitute for breast surgery procedures, such as lumpectomy. Imaging technologies are crucial to localize the target area and to assess the treatment effect during and after ablation treatment. US is most valuable in providing the location of the electrode within the lesion and in real-time monitoring the ablation procedures, but it can not obtain the information about the spatial structure of the tumor. So US can not accurately assess the efficacy ablative therapy. MRI is the most optimal modality for visually describing the tumor volume and for evaluating the ablative efficacy. In recent years, several ablation techniques, including radiofrequency ablation (RFA), laser ablation, cryoablation and microwave ablation (MWA) have been evaluated in clinical trials (2-6). Compared with others ablation techniques, MWA is a promising technique because it can preferentially heat and damage high-watercontent breast tumors, whereas the lesser degrees of heating lower-water-content fatty and glandular tissues. Currently, percutaneous MWA is being increasingly used for treating solid tumors, such as liver, lung, and kidney tumors, to our knowledge, there is few studies reporting the clinical efficacy of microwave ablated breast tumor. In the present study, we analyzed gray-scale and enhanced US features of 205 microwave ablated breast benign lesions and compared with MRI and histopathology findings, to discuss the safety and efficiency of US-guided MWA ablation in the treatment of breast benign tumor patients.

\section{Methods}

We obtained written informed consent from all the subjects involved in the study and the University Ethics Committee approved this prospective study. Between October 2013 and December 2016, percutaneous MWA was performed following conventional gray-scale US in 182 consecutive patients at our hospital, who underwent evaluation of breast lesions according to the breast US lexicon: breast imaging reporting and data system (BI-RADS). The symptoms of patients included pain, nipple discharge and mental stress. We selected 205 BI-RADS 2 and 3 breast lesions (mean size $14.41 \pm 6.54 \mathrm{~mm}$; range, $5.6-30.0 \mathrm{~mm}$ ) from those as the final study group. All of the patients are women, mean age $37.6 \pm 11.4$ (range, 16-69) years. Inclusion criteria: the lesion located in the breast gland confirmed by preoperative US; the distance between the lesion and the skin and pectoral fascia are more than 1 centimeter and be reason of cosmetic requirement or physical condition refused to or not tolerated surgical resection. Exclusion criteria: patient with serious coagulation disorders; patient with acute or active infectious diseases; patient with serious hypertension, diabetes and cardiopulmonary dysfunction; the lesion is more than 3 centimeters in diameter and pregnancy.

Conventional US, contrast-enhanced US (CEUS) and WMA were performed with HI VISION Ascendus (Hitachi Medical, Tokyo, Japan) US equipment with a linear probe (L74M). The microwave system (Nanjing Yigao Microwave Institute, Nanjing, China) consisted of a microwave generator and a hollow water-cooled-shaft antenna (with a 3 -millimeter long front-end of microwave transmission), a flexible coaxial cable and a microwave generator. Before the ablation therapy, the lesions met with one of the following criteria, the largest diameter $>20 \mathrm{~mm}$ or grade II/III blood flow were performed enhanced MRI. The MRI system used was the MAGNETOM Verio 3.0T (Siemens, Erlangen, Germany) in our study. Gadopentetate dimeglumine (Consun, Guangzhou, China) was used as a contrast agent.

The feature of gray-scale US of the lesion was described using BI-RADS sonographic descriptors, including shape (oval, round, irregular), longitudinal/transverse (L/T, $>1, \leq 1)$, boundary (ill-defined, well-defined), margin (circumscribed, lobulated, indistinct), echogenicity (hyperechoic, iso-echoic, hypo-echoic, complex) and posterior acoustic features (enhancement, no change, attenuation). The largest diameter and volume of the lesions were obtained also.

Then, CEUS performed on the lesion. Standard machine settings were used, with mechanical index 0.05 to 0.08 , and the focus was placed deeper than the lesion plane so as not to destroy the microbubbles. SonoVue (BR1, Bracco Imaging, Milan, Italy) was used as a contrast agent, which was supplied as a sterile, lyophilised powder contained in a septum-sealed vial. A suspension of the contrast agent was obtained by adding $5 \mathrm{~mL}$ of physiological saline to the powder $(25 \mathrm{mg})$. A contrast bolus of $5 \mathrm{~mL}$ was injected manually at a flow of approximately $1 \mathrm{~mL} / \mathrm{s}$, followed by a 5 -mL saline solution flush, via a 20 -gauge intravenous cannula placed in an antecubital vein by a nurse. Using an Time-triggered acquisition over a period of $120 \mathrm{~s}$, the imaging data obtained from CEUS were continuously stored by an integrated magnetic optical drive on the US machine. The intralesional and perilesional enhancement intensity were observed. Finally, the lesions were undergone US-guided fine-needle aspiration biopsy (US-FNA).

US-guided percutaneous MWA performed on the lesion that confirmed as benign by US-FNA. In this study, 
Table 1 Comparison of the longest diameters of breast lesions before and after MWA

\begin{tabular}{lcc}
\hline MWA & $\mathrm{n}$ & Longest diameter \\
\hline Before MWA & 205 & $14.41 \pm 6.54$ \\
$1 \mathrm{~h}$ after MWA & 205 & $18.56 \pm 7.33$ \\
3 m after MWA & 202 & $12.16 \pm 6.54$ \\
6 m after MWA & 191 & $9.52 \pm 6.16$ \\
12 m after MWA & 161 & $8.48 \pm 6.30$
\end{tabular}

MWA, microwave ablation.

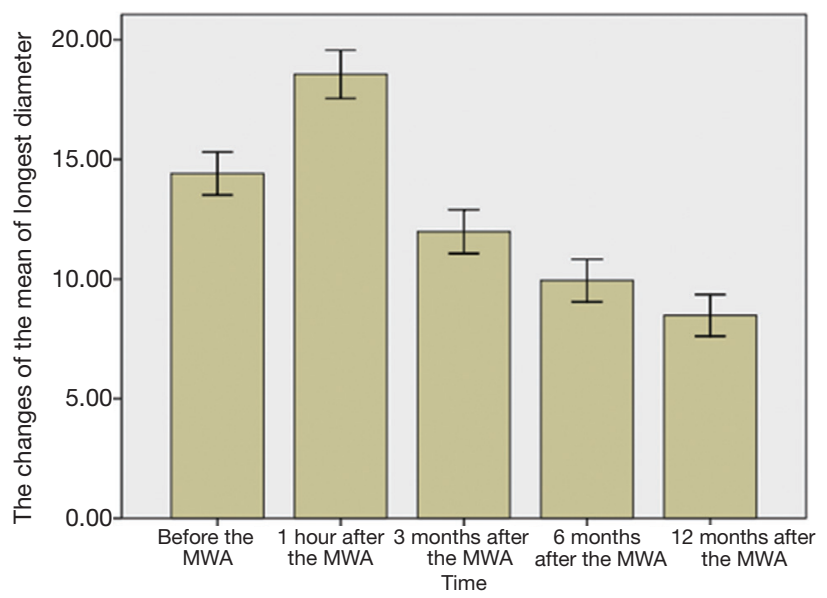

Figure 1 The histogram showed the mean of the longest diameter of all ablative lesions was increased $1 \mathrm{~h}$ after ablation and it was gradually decreased 3, 6 and 12 months after the ablation.

an output power of $40 \mathrm{~W}$ and an electromagnetic field of 2.5 $\mathrm{MHz}$ were selected when implemented the WMA. According to lesion's location, shape, size and the findings of preoperative US and/or MRI, designed a reasonable ablation scheme, such as the duration time and times of the ablation, the penetrated orientation of the needle (the distribution of ablation electrode) and the range of the ablation, etc. The patient was positioned supine or latericumbent and sterilized and local anesthesia. If the distance between the lesion and the skin and/or pectoral fascia is less than 1 centimeter, we injected $5 \%$ saline as a spacer into the subcutaneous and/or retromammary space to widen the distance between them. Subsequently, a skin incision was made and the water-cooled-shaft antenna was introduced percutaneously under US guidance. After the tip of the antenna was in place, started the ablation with real-time US monitoring. The duration of microwave irradiation was between 3-8 min, over which almost all of the tumor was ablated. During the process of ablation, a nurse monitored the temperature at the tip of the antenna on the instrument panel of the microwave system and the complications appeared in the patients. Then, the same procedure was repeated in other lesions if more than one lesion underwent the MWA. At the end of MWA, the generator was turned off and the water-cooled-shaft antenna was removed.

One hour after the MWA, CEUS performed on the lesions to observe and evaluate the efficiency of the MWA. If the intralesional and/or perilesional area presented the enhancement on CEUS, we performed further supplementary ablation and CEUS again. After these procedures, enhanced MRI performed on the lesion to further assess therapeutic efficiency, evaluated parameters included the lesion volume, intralesional and perilesional enhancement intensity. The follow-up was implemented 3, 6 and 12 months after the MWA by using gray-scale US and CEUS examinations to evaluate the long-term efficacy of ablative lesions. Six months after the ablation, enhanced MRI was selectively performed on the ablative lesions. The evaluation parameters included: whether the lesions ablated completely or not (presence or absence of residual and recurrence); the change of the size of the lesions (no-change, decreased of disappeared); the improvement of clinical symptoms (relieved or disappeared); cosmetic outcome (presence or absence of scar, asymmetry or symmetry of bilateral breast) and cost-efficiency ratio.

SPSS 17.0 for Windows ${ }^{\circledR}$ software package (SPSS Inc., Chicago, IL, USA) was used for statistical analysis. We compared the size of the lesions before and after the MWA by using the single factor variance analysis. $P$ values smaller than 0.05 was considered statistical significance.

\section{Results}

The breast nodules confirmed by US-FNA were fibroadenoma in 103, intraductal papilloma in 11, hyperplastic lesions in 51 and adenosis in 40, respectively. The longest diameter of the ablative lesion was increased one hour after MWA, however, it was gradually decreased, even disappeared 3, 6 or 12 months after MWA (Table 1, Figure 1). During the follow-up, the volume of the ablative lesion was gradually decreased and 44 (/205, 21.5\%) ablative lesions were disappeared 12 months after the MWA (Table 2, Figures 2,3). The success rate of the MWA treatment in the benign breast lesion was $82.93 \%$. Moreover, the 
Table 2 Comparison of the volume of breast lesion before and after MWA

\begin{tabular}{lcc}
\hline MWA & $\mathrm{n}$ & Lesional volume $\left(\mathrm{mm}^{3}\right)$ \\
\hline Before MWA & 205 & $3,224 \pm 961$ \\
3 months after MWA & 202 & $2,909 \pm 872$ \\
6 months after MWA & 191 & $2,634 \pm 815$ \\
12 months after MWA & 161 & $2,116 \pm 732$ \\
F & & 3.921 \\
P & & 0.01 \\
\hline
\end{tabular}

MWA, microwave ablation.

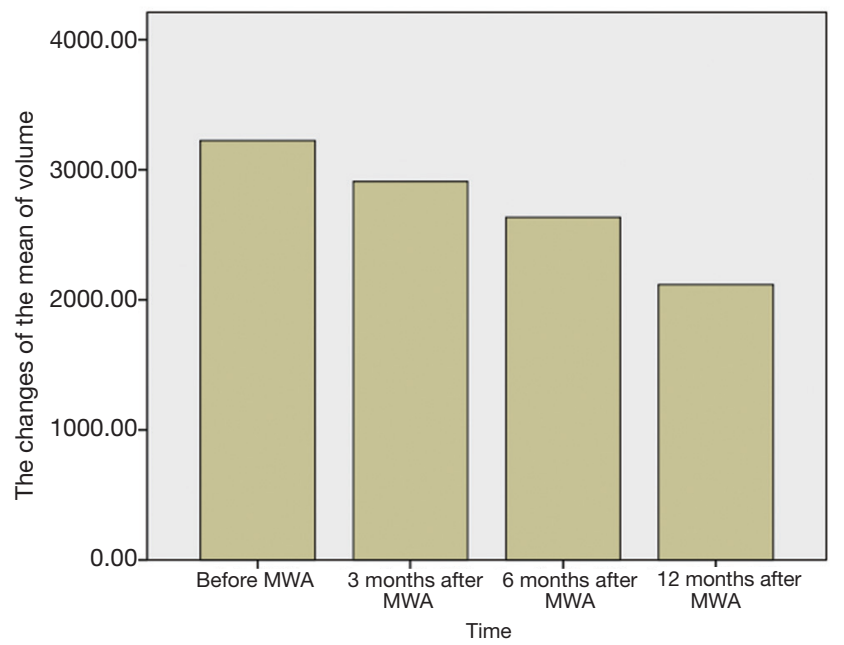

Figure 2 The histogram showed the mean of the volume of all ablative lesions was increased $1 \mathrm{~h}$ after ablation and it was gradually decreased 3, 6 and 12 months after the ablation.

symptoms of patient were relieved or disappeared after the MWA.

Before the MWA, the breast lesion presented a hypoechoic mass with a well-defined boundary in 187 (/205, 91.2\%) lesions. During the ablation, all ablative lesions presented a hyperechoic mass with an ill-defined boundary. One hour after the MWA, all lesions presented a heterogeneous hypoechoic mass and the boundary of them gradually became clearly. Of those, $69.3 \%(142 / 205)$ ablative lesions presented a hypoechoic halo surrounding it on gray-scale US one hour after the MWA.

Before performing MWA, 182 (/205, 88.8\%) lesions presented various degrees of enhancement on CEUS. One hour after the MWA, the enhanced feature of the ablative lesions both on CEUS and enhanced MRI was shown in Table 3. For 26 enhanced lesions, punctate or 1-2 linear enhancements appeared in the perilesional region. According to the enhanced MRI, 9 lesions which presented no-enhancement on CEUS showed the enhancement appeared in the perilesional region. A total of 35 lesions were undergone further supplementary ablation. After the ablation procedure, enhanced MRI showed that there had no obvious enhancement within the lesions.

During the ablation, no patient had serious complications, such as hemorrhage, serious pain and fat necrosis, etc. Nine cases presented duct ectasia located in the peripheral region of the lesion after MWA, but nipple discharge did not appear in all cases, which disappeared after the symptomatic treatment. Fifteen cases presented slightly local pain and skin erythroswelling during or after the ablation and the symptom relieved within 8-12 hours after the physiotherapy. Four cases presented local fat liquefaction, which recovered after aspirating liquid under US-guidance. All cases did not present secondary infection.

\section{Discussion}

There is increasing interest in the use of minimally invasive ablative techniques in the treatment of breast tumor. Image-guided nonsurgical ablative therapies, including radiofrequency, MWA and high-intensity focused US have received increasing attention as minimally invasive treatments to destroy various solid tumors. The breast is the perfect organ for ablation because the breast locates in superficial location, cover only by skin with no intervening structures, can be compressed asymmetrically to achieve ablation strategy and monitored effectively by US (6). MWA is a promising minimally invasive local therapy with many advantages, including large ablation zones, short ablation durations, and improved convection profile (7-9). Currently, MWA has been explored in clinical practice, which destroys tumors in situ by means of thermal coagulation or protein denaturation (10). Several studies reported successful experience in the treatment of small breast cancer by using MWA (11,12).

Before the ablation, precise determination of lesion's position and accurate measurement of its volume was the key to ensure completed ablation, as well as understanding the space structure and the relationship between the lesion and the surrounding tissue. Although US can accurately assess the features of lesional size, morphology and blood flow, it can not obtain the information about the spatial 

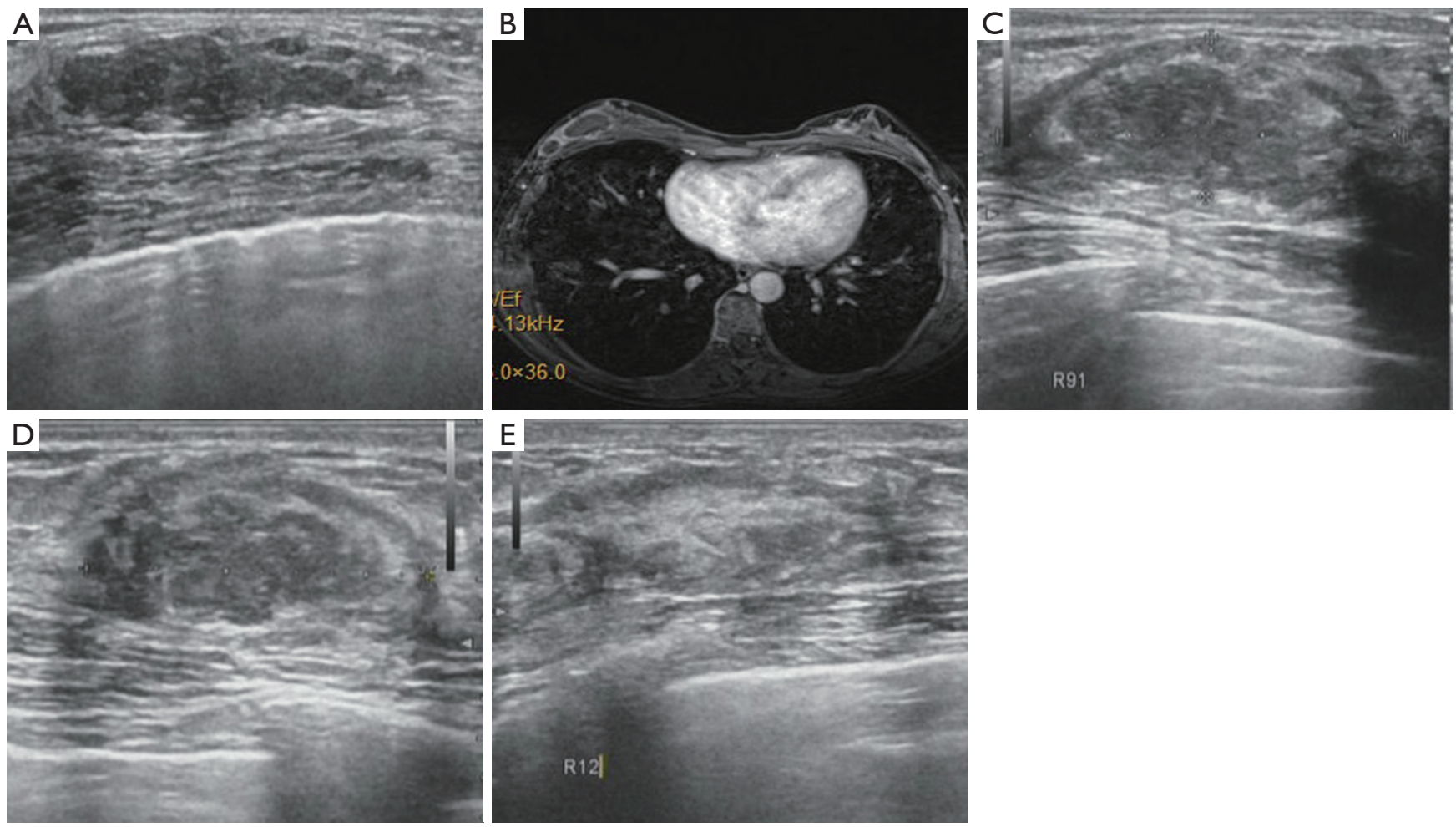

Figure 3 Images of a 38-year-old woman with fibroadenoma which was classified BI-RADS 3. (A) Gray-scale US presented a hypoechoic, well-defined boundary, solid mass with a transverse axis diameter of $30 \mathrm{~mm}$ located at the right breast; (B) after the MWA, MRI showed the lesion had no enhanced and the lesional periphery had an "edema-zone"; (C) three months after the MWA, gray-scale US the lesion was decreased with a largest diameter of $24 \mathrm{~mm}$, and the lesional periphery had a hypoechoic halo corresponding to the "edema-zone" on MRI; (D) the image showed the lesion was further decreased with a largest diameter of $19 \mathrm{~mm}$ six months after the MWA; (E) the gray-scale image showed that the lesion was almost disappeared 12 months after the MWA. MWA, microwave ablation; MRI, magnetic resonance imaging.

Table 3 Comparison of CEUS and enhanced MRI evaluating ablative efficacy $1 \mathrm{~h}$ after the MWA

\begin{tabular}{lccc}
\hline \multirow{2}{*}{ Imaging method } & \multicolumn{2}{c}{ Enhanced feature } & Success rate \\
\cline { 2 - 3 } & No-enhancement & Enhancement & $87.32 \%(179 / 205)$ \\
CEUS & 179 & 26 & $82.93 \%(170 / 205)$ \\
Enhanced MRI & 85 & 9 & 8 \\
\hline
\end{tabular}

CEUS, contrast-enhanced ultrasound; MRI, magnetic resonance imaging; MWA, microwave ablation.

structure of the lesion because of its poor spatial resolution. So US can not accurately measure the volume of the lesion and not accurately observe the spatial distribution of lesional blood flow (13). Breast MRI is the most sensitive examination for breast tumor detection and it can be used to detect the spatial relationship between the lesion and the surrounding tissue, the spatial distribution of lesional blood flow as well (14-18). What's more, MRI can be used to assess tissue necrosis after ablation by administrating contrast agent $(19,20)$. So MRI is suitable for evaluating the efficacy of MWA. In this study, we designed the ablation strategies according to information about the volume and vascular distribution of lesion provided by CEUS and/ or enhanced MRI. The results showed that 170 of 205 lesions were complete ablation, which was presented noenhancement in both intralesional and perilesional area 
on CEUS and/or MRI after the ablation. Of 176 lesions were no enhanced on CEUS, 9 lesions presented the enhancement in the perilesional area on enhanced MRI. Therefore, enhanced MRI was superior to CEUS in the detection of the spatial distribution of the vessels within the lesion.

One hour after the MWA, US and/or MRI showed the largest diameter of the ablative lesions were larger than that of the lesions before the ablation. In addition, a hypoechoic halo appeared in the periphery of the ablative, which was defined as a "edema-zone". The reasons of which may be that under the action of high temperature, the ablated tissues appear in carbonization and coagulative necrosis, accompanying fibrous connective tissue hyperplasia, inflammatory reaction and cellular edema, etc., which made the size of the lesion increase and the hypoechoic halo appear in the periphery of the lesion on US. During the follow-up, the "edema-zone" was gradually disappeared or became blurred. However, in some cases, the hypoechoic halo was not disappeared. So we performed US-FNA to understand the histological components of the hypoechoic halo and the histopathology showed that the reasons on the formation of it was due to the coagulative necrosis and collagen of the surrounding normal tissues. Therefore, we should systematically evaluate the spatial position and blood flow of the lesion and design an optimal ablative strategies to avoid the injury of surrounding tissues as much as possible.

The pathological confirmation of the efficacy is the gold standard for MWA of breast tumor. CEUS can evaluate the ablative efficacy by observing the vascular enhancement after the administration of contrast agent. However, because of the influence of the heat generated after the ablation, CEUS was difficult in the detection of the perilesional area. Enhanced MRI would not influence by the heat, it can achieve the stereo observation of the intralesional vessel distribution. In our study, all ablative lesions were performed US-FNA after the MWA. Both histopathology and/or enhanced MRI showed that all ablative lesions presented various degrees of coagulative necrosis, there had the correlation between MRI and histopathological findings.

That the incidence of the recurrence and local complications must be minimal was the important index for assessing the long-term efficacy of ablative therapy. In our series, no patient had the recurrence and the largest diameter and volume of all ablative lesions were gradually decreased during the follow-up of one year.
During and after the ablation, local complications, such as mild pain, skin redness and swelling, duct dilation and fat liquefaction was appeared in $69(/ 182,38 \%)$ cases, which was relieved or disappeared after the symptomatic treatment.

There were two main limitations in our study. Firstly, we selectively performed MRI on the ablative lesions to evaluate the efficacy after the MWA and the findings showed the efficacy was satisfied. However, the majority of lesions only performed CEUS to evaluate the efficacy after the MWA, the long-term efficacy of these lesions remains to be observed. Secondly, the mean largest diameter of the lesion in our study was $14.4 \mathrm{~mm}$, only 75 (36.6\%) of 205 lesions was more than $15 \mathrm{~mm}$ in largest diameter. For larger lesions $(20 \mathrm{~mm}<$ largest diameter $<30 \mathrm{~mm})$, the efficacy of US-guided MWA need further discussion in the future.

\section{Conclusions}

MWA was a safe and efficient method for the treatment of the benign breast tumors. CEUS combined with enhanced MRI could clearly exhibited the morphological and pathological changes of the lesion after the MWA. In contrast to the histopathological findings, they can efficiently assess the efficacy of microwave ablative breast benign lesions and be necessary for long-term follow-up.

\section{Acknowledgements}

None.

\section{Footnote}

Conflicts of Interest: The authors have no conflicts of interest to declare.

Ethical Statement: We obtained written informed consent from all the subjects involved in the study and the University Ethics Committee approved this prospective study (No. 2013006).

\section{References}

1. Vargas HI, Dooley WD, Gardner RA, et al. Focused microwave phased array thermotherapy forablation of early-stage breast cancer: results of thermal dose escalation. Ann Surg Oncol 2004;11:139-46. 
2. Brkljacic B, Cikara I, Ivanac G, et al. Ultrasoundguided bipolar radiofrequency ablation of breast cancer in inoperable patients: a pilot study. Ultraschall Med 2010;31:156-62.

3. Grotenhuis BA, Vrijland WW, Klem TM. Radiofrequency ablation for early-stage breast cancer: treatment outcomes and practical considerations. Eur J Surg Oncol. 2013;39:1317-24.

4. Furusawa H, Namba K, Thomsen S, et al. Magnetic resonance-guided focused ultrasound surgery of breast cancer: reliability and effectiveness. J Am Coll Surg 2006;203:54-63

5. Waaijer L, Kreb DL, Fernandez Gallardo MA, et al. Radiofrequency ablation of small breast tumours: evaluation of a novel bipolar cool-tip application. Eur J Surg Oncol 2014;40:1222-9.

6. Xu X, Luo L, Chen J, et al. Acoustic radiation force impulse elastography for efficacy evaluation after hepatocellular carcinoma radiofrequency ablation: a comparative study with contrast-enhanced ultrasound. Biomed Res Int 2014;2014:901642.

7. Simon CJ, Dupuy DE, Mayo-Smith WW. Microwave ablation: principles and applications. Radiographics 2005;25:S69-83.

8. Yu J, Liang $\mathrm{P}, \mathrm{Yu} \mathrm{X}$, et al. A comparison of microwave ablation and bipolar radiofrequency ablation both with an internally cooled probe: results in ex vivo and in vivo porcine livers. Eur J Radiol 2011;79:124-30.

9. Zhou W, Liang M, Pan H, Liu X, Jiang Y, Wang Y, et al. Comparison of ablation zones among different tissues using $2450-\mathrm{MHz}$ cooled-shaft microwave antenna: results in ex vivo porcine models. PLoS One 2013;8:e71873.

10. Zhou W, Wang R, Liu X, et al. Ultrasound-guided microwave ablation: a promising tool in management of benign breast tumours. Int J Hyperthermia 2016;10:1-8.

11. Zhou W, Zha X, Liu X, et al. US-guided percutaneous microwave coagulation of small breast cancer: a clincal

Cite this article as: Zhang W, Li JM, He W, Pan XM, Jin ZQ, Liang T, Zhang H. Ultrasound-guided percutaneous microwave ablation for benign breast lesions: evaluated by contrastenhanced ultrasound combined with magnetic resonance imaging. J Thorac Dis 2017;9(11):4767-4773. doi: 10.21037/ jtd.2017.09.132 study. Radiology 2012;263:364-73.

12. Zhu M, Lin XA, Zha XM, et al. Evaluation of the therapeutic efficacy of sequential therapy involving percutaneous microwave ablation in combination with 131I-hypericin using the VX2 rabbit breast solid tumor model. PLoS One 2015;10:e0120303.

13. Sag AA, Maybody M, Comstock C, et al. percutaneous image-guided ablation of breast tumors: an overview. Semin Intervent Radiol 2014;31:193-202.

14. Yuan J, Wong OL, Lo GG, et al. Statistical assessment of bi-exponential diffusion weighted imaging signal characteristics induced by intravoxel incoherent motion in malignant breast tumors. Quant Imaging Med Surg 2016;6:418-29.

15. Lee SC, Grant E, Sheth P, et al. Accuracy of ContrastEnhanced Ultrasound Compared With Magnetic Resonance Imaging in Assessing the Tumor Response After Neoadjuvant Chemotherapy for Breast Cancer. J Ultrasound Med 2017;36:901-11.

16. Eghtedari M, Ma J, Fox P, Guvenc I, Yang WT, Dogan BE. Effects of magnetic field strength and $b$ value on the sensitivity and specificity of quantitative breast diffusionweighted MRI. Quant Imaging Med Surg 2016;6:374-80.

17. Giess CS, Chikarmane SA, Sippo DA, et al. Clinical Utility of Breast MRI in the Diagnosis of Malignancy After Inconclusive or Equivocal Mammographic Diagnostic Evaluation. AJR Am J Roentgenol 2017;208:1378-85.

18. Ha R, Mema E, Guo X, et al. Quantitative 3D breast magnetic resonance imaging fibroglandular tissue analysis and correlation with qualitative assessments: a feasibility study. Quant Imaging Med Surg 2016;6:144-50.

19. Pinker K, Helbich TH, Morris EA. The potential of multiparametric MRI of the breast. Br J Radiol 2017;90:20160715.

20. Lohrke J, Frenzel T, Endrikat J, et al. 25 Years of ContrastEnhanced MRI: Developments, Current Challenges and Future Perspectives. Adv Ther 2016;33:1-28. 